\title{
Assessment of Lesion Response in the Initial Radioiodine Treatment of Differentiated Thyroid Cancer Using ${ }^{124}$ I PET Imaging
}

\author{
Walter Jentzen ${ }^{1}$, Jan Hoppenbrouwers ${ }^{2}$, Paul van Leeuwen ${ }^{2}$, Daan van der Velden², Rudie van de Kolk², \\ Thorsten Dirk Poeppel ${ }^{1}$, James Nagarajah ${ }^{1}$, Wolfgang Brandau ${ }^{1}$, Andreas Bockisch ${ }^{1}$, and Sandra Rosenbaum-Krumme ${ }^{1}$ \\ ${ }^{1}$ Klinik für Nuklearmedizin, Universität Duisburg-Essen, Essen, Germany; and ${ }^{2}$ Fontys University of Applied Sciences, Eindhoven, \\ The Netherlands
}

\begin{abstract}
${ }^{124}$ I PET/CT images from differentiated thyroid cancer patients were retrospectively analyzed to assess the relationship between absorbed radiation dose $(A D)$ to lesions and their response after radioiodine therapy. Methods: Patients received serial ${ }^{124}$ I PET/CT scans before and after their first radioiodine treatment. The pretherapy PET data were used to segment the lesion volumes and to predict the therapy-delivered ADs after administration of the therapeutic ${ }^{131}$ | activity. The segmentation method's lower volume limit of determinability was a sphere of $0.80 \mathrm{~mL}$, which classified the lesions into a known-volume group ( $>0.80 \mathrm{~mL}$ ) or a small-volume group $(\leq 0.80 \mathrm{~mL})$ with their respective average and minimum ADs. The posttherapy PET data were used to assess the lesion-based therapy success. In the known-volume group, the response rate was calculated on the basis of lesions that received average $A D s$ above the generally accepted threshold of 85 Gy for metastases and 300 Gy for thyroid remnants (TRs) and was expressed as the percentage of completely responding lesions. In the small-volume group, the metastasis and TR responses were evaluated for 3 minimum-AD groups: 5 to $10 \mathrm{~Gy}$ (TR, 5 to $30 \mathrm{~Gy}$ ), $>10$ to $85 \mathrm{~Gy}$ (TR, $>30$ to $300 \mathrm{~Gy}$ ), and $>85 \mathrm{~Gy}$ (TR, $>300$ Gy). Their response rates were calculated in terms of the percentage of completely responding lesions in each minimum-AD group. Results: In total, 59 lesions in 17 patients were amenable to reliable volume estimation. The response rates were $63 \%, 88 \%$, and $90 \%$ for lymph node metastases (LMs), pulmonary metastases, and TRs, respectively. The response rates of 168 small lesions in 34 patients were more than $82 \%$ for LMs and more than $91 \%$ for TRs in each of the 3 minimum-AD groups; all small pulmonary metastases responded completely. Conclusion: In the known-volume group, the response rate for TRs matched well with historical data derived using ${ }^{131}$ I scintigraphy imaging, whereas the response rate for LMs was not as high as expected, which may be explained by too short a follow-up time for a few LMs and a higher sensitivity of PET imaging. Small lesions were treated effectively, suggesting that they are considerably smaller than $0.80 \mathrm{~mL}$.
\end{abstract}

Key Words: thyroid carcinoma; dosimetry; ${ }^{124}$; radioiodine therapy

J Nucl Med 2014; 55:1759-1765

DOI: 10.2967/jnumed.114.144089

Received Jun. 5, 2014; revision accepted Sep. 8, 2014.

For correspondence or reprints contact: Walter Jentzen, Klinik für Nuklearmedizin, Universität Duisburg-Essen, Hufelandstrasse 55, D-45122 Essen, Germany.

E-mail: walter.jentzen@uni-duisburg-essen.de

Published online Oct. 20, 2014.

COPYRIGHT (c) 2014 by the Society of Nuclear Medicine and Molecular Imaging, Inc.
$\mathbf{R}$ of differentiated thyroid cancer (DTC) after total thyroid (except for patients at very low risk, such as those with papillary microcarcinoma). Radioiodine treatment is used primarily to eliminate tumor tissue, that is, locoregional lymph node metastases (LMs), distant metastases such as pulmonary metastases (PMs) and bone metastases (BMs), and secondarily to ablate thyroid remnants (TRs). In particular, the successful treatment of metastases is relevant because patients with metastases that completely respond have considerably higher survival rates than patients with incompletely responding metastases (1).

The established quantity of radiation that mediates successful therapy is the absorbed radiation dose (AD) to lesions. Historically, the relationship between $\mathrm{AD}$ and response success was investigated using follow-up whole-body planar scintigraphy with ${ }^{131}$ I. The milestone publications remain the tracer dosimetry studies by Maxon et al. $(2,3)$ even after more than 2 decades. They demonstrated that an $\mathrm{AD}$ threshold of at least $85 \mathrm{~Gy}$ for LMs and 300 Gy for TR ablation was associated with complete response rates of approximately $80 \%-90 \%$. These AD thresholds are ubiquitous and are often cited in guidelines $(4,5)$. They may play a pivotal role in the selection of a suitable amount of therapeutic ${ }^{131}$ I activity when pretherapy lesion dosimetry is performed. Over the last decade, pretherapy dosimetry using ${ }^{124}$ I PET has become more frequent (6-8). Compared with ${ }^{131}$ I scintigraphy, ${ }^{124}$ I PET has a higher sensitivity for lesion detection (9) and a higher quantitative capacity (10). Using pretherapy ${ }^{124}$ I PET imaging, our group estimated the AD per administered activity in several studies. These data, in addition to other key factors, were used to optimize the amount of a single ${ }^{131}$ I activity. Maxon's AD thresholds were part of the decision-making process.

Since 2003, our department has been routinely conducting pretherapy ${ }^{124}$ I PET/CT dosimetry for high-risk DTC patients. Several patients received a second postherapy ${ }^{124}$ I PET scan as part of the follow-up examination to prepare for the second radioiodine therapy. These data offer the potential to assess the complete response above the accepted AD thresholds using ${ }^{124}$ I PET. The preevaluation of a plethora of PET/CT data revealed that there were 2 groups of lesions. In the first known-volume group, reliable volume estimations were possible using an established PET-based thresholding method (11). The second group contained lesions with imprecise volume estimations; that is, the volumes were too small or were below the thresholding method's limit of determinability of $0.80 \mathrm{~mL}$ (small-volume group). Consequently, the 
actual ADs of these small lesions cannot be reliably determined; however, a fixed volume of $0.80 \mathrm{~mL}$ can be used in the AD calculation that will yield a minimum AD. Thus, the aim of this study was to assess the therapy response above the accepted $\mathrm{AD}$ thresholds of lesions in the known-volume group and to explore the relationship between therapy response and minimum $\mathrm{AD}$ of small lesions.

\section{MATERIALS AND METHODS}

\section{Patient Collective and Preparation}

We retrospectively analyzed the data of DTC patients who met both patient and lesion inclusion criteria. Patients were included who received both ${ }^{124}$ I PET/CT dosimetry before their first radioiodine therapy and posttherapy ${ }^{124}$ I PET/CT dosimetry. The posttherapy dosimetry data were used to plan a second radioiodine therapy and were also part of the follow-up examination. These patients had not received any treatments such as surgery or external-beam radiation therapy in the time period after the radioiodine treatment until the follow-up ${ }^{124}$ I PET scans. All patients had histologically confirmed advanced DTC (papillary or follicular). Two main lesion inclusion criteria were applied. The unambiguous tissue assignment as either TR or LM was performed using localization criteria (12). If the assignments were doubtful, the lesions were excluded. The lesions had to be sufficiently separated and distant from radioiodine-accumulating tissues to minimize spill-in of external activity into the lesion.

All patients underwent total thyroidectomy. Patient preparation was similar for the pretherapy and follow-up dosimetry. The patients were either under endogenous thyroid-stimulating hormone (TSH) stimulation (by levothyroxine withdrawal for $4 \mathrm{wk}$ ) or under exogenous TSH stimulation (by use of recombinant human TSH), with TSH levels of at least $30 \mathrm{mIU} / \mathrm{L}$. The patients were instructed to be on a low-iodine diet for $4 \mathrm{wk}$ before the ${ }^{124} \mathrm{I}$ PET/CT scans. Iodine contamination was checked by urine testing. Serial PET/CT scans were acquired after the administration of approximately $25 \mathrm{MBq}$ of ${ }^{124} \mathrm{I}$. The single therapeutic ${ }^{131} \mathrm{I}$ activity was individually assessed by an expert team that considered the pretherapy results for all lesions as well as the results for the toxicity levels of the activity-limiting organs: bone marrow and lung (6). The treatment activity was administered $1-2 \mathrm{~d}$ after the last pretherapy scan. The follow-up PET scan was performed typically between 3 and 6 mo, according to the German guidelines (13). The patients provided written informed consent to undergo the examination, and the study was approved by the local ethics committee.

\section{Image Acquisition and Reconstruction}

The images were acquired on a Biograph Duo PET/CT scanner (Siemens Medical Solutions). The pretherapy and follow-up images acquired at 24 and at least $96 \mathrm{~h}$ after ${ }^{124} \mathrm{I}$ administration were retrospectively analyzed using a reliable simplification of the comprehensive 5-point protocol (14). The PET/CT scans were acquired from head to thigh, beginning with spiral CT (tube voltage of $110 \mathrm{kVp}$, tube current time product of $15 \mathrm{mAs}$, a pitch of 1.6, and a slice thickness of $5 \mathrm{~mm}$ ). The PET emission time was $300 \mathrm{~s}$ per bed position. No contrast agent was applied.

The PET images were retrospectively reconstructed using parameters optimized for quantitative ${ }^{124} \mathrm{I}$ imaging (15). The emission images were reconstructed after Fourier rebinning using the iterative attenuation-weighted ordered-subset expectation maximization algorithm with 4 iterations and 16 subsets. A postreconstruction gaussian smoothing filter of $5 \mathrm{~mm}$ was applied. Standard scatter, attenuation, and dead-time corrections provided by the manufacturer were used. The reconstructed transverse emission images had a voxel size of $1.7 \times$ $1.7 \times 2.4 \mathrm{~mm}^{3}$. The reconstructed PET spatial resolution was $8.2 \mathrm{~mm}$. The CT image was reconstructed using a reconstruction kernel B40s and had a voxel size of $1.0 \times 1.0 \times 2.4 \mathrm{~mm}^{3}$.

\section{Functional Volumes and Classification into Known- and Small-Volume Groups}

CT or ultrasonography images are commonly used to determine lesion volumes. CT images could have been used; however, discerning the lesions was often difficult or not possible primarily because no iodinated contrast agent could be used in a CT acquisition before radioiodine therapy. Only ultrasonography can be used for most of the cervical LMs, and postoperative delineation of TRs is problematic. To include all lesions observed on pretherapy ${ }^{124}$ I PET images and to use a consistent methodology, an established PET-based image-thresholding method was applied (11) to pretherapy ${ }^{124}$ I PET images $24 \mathrm{~h}$ after ${ }^{124} \mathrm{I}$ administration. The segmentation method's lower volume limit of determinability was a sphere of $0.80 \mathrm{~mL}$ (11.5 $\mathrm{mm}$ in diameter), which classified the lesions into 2 groups. The first group was amenable to reliable volume estimations (known-volume group, $>0.80 \mathrm{~mL}$ ); the second group comprised smaller lesions (small-volume group, $\leq 0.80 \mathrm{~mL}$ ).

\section{Average and Minimum ADs in Therapy}

The AD calculation involved different steps and has been described in previous studies $(10,16)$. Average ${ }^{124}$ I activity concentrations were evaluated for lesions in the known-volume group, whereas ${ }^{124} \mathrm{I}$ activity concentrations using a spheric VOI with a fixed volume of $0.80 \mathrm{~mL}$ were ascertained for the lesions in the small-volume group. In followup images, the volumes often decreased below $0.80 \mathrm{~mL}$; in that case, the fixed-volume activity concentration was used to estimate uptake. (A percentage ${ }^{124} \mathrm{I}$ uptake of approximately $0.001 \% / \mathrm{mL}$ can be detected using a tracer ${ }^{124} \mathrm{I}$ activity of $25 \mathrm{MBq}$, provided that the background is sufficiently low.)

Two corrections, partial-volume effect and half-life, were necessary to obtain the projected ${ }^{131}$ I activity concentrations and their uptake values, which were used to estimate the ${ }^{131}{ }^{1}$ residence time. Using the ${ }^{131} \mathrm{I}$ uptake values at $24 \mathrm{~h}$ and at least $96 \mathrm{~h}$, either a biphasic or a triphasic model was applied to estimate the residence time. The choice of the model depended on the value of the effective ${ }^{131}$ I half-life. When the effective ${ }^{131}$ I half-life was less than or equal to the physical ${ }^{131}$ I half-life, the residence time was estimated using the adapted 2-point approach (14). In this approach, the uptake curve was biphasic and was parameterized using a combination of a linear function and a monoexponential function. The uptake curve was parameterized with a triphasic model when the effective ${ }^{131}$ I half-life was greater than the physical ${ }^{131}$ I half-life (only $\sim 10 \%$ of the lesions evaluated). The residence time arising from the first and second phases was the time integral from zero to the last measured point using the functions based on the biphasic model. The contribution resulting from the third phase was calculated assuming physical decay after the last imaging time point.

This procedure yielded average and minimum ADs per administered activity for known-volume and small-volume lesions, respectively. In the AD calculation, the tissue densities of $1.04 \mathrm{~g} / \mathrm{mL}$ (LM, $\mathrm{PM}$, and TR) and $1.3 \mathrm{~g} / \mathrm{mL}(\mathrm{BM})$ were used. For each lesion, the predicted average and minimum ADs in therapy were calculated using the single therapeutic activity. A more rapid ${ }^{131}$ I release is expected from damaged cells during therapy; therefore, the PET-based ADs were slightly higher than the actually delivered ADs in therapy. In addition, stunning may diminish the ADs in therapy (17); however, because the administered tracer ${ }^{124}$ I activity in this study was relatively low $(\sim 25 \mathrm{MBq})$, stunning was not likely to have a significant impact on therapeutic efficacy $(13,17)$.

\section{Minimum-AD Groups}

The small lesions were divided into 3 minimum-AD groups. The minimum-high-AD group comprised lesions above 85 Gy for metastases and $300 \mathrm{~Gy}$ for TRs. The dividing lines between the minimumlow- and minimum-mid-AD groups were approximately one tenth of 85 Gy for metastases and 300 Gy for TRs. Hence, the minimum-low- and minimum-mid-AD groups contained metastases with minimum ADs 
of 5 to $10 \mathrm{~Gy}$ (TR, 5 to $30 \mathrm{~Gy}$ ) and $>10$ to $85 \mathrm{~Gy}$ (TR, $>30$ to 300 Gy), respectively.

\section{Response Index and Response Rate}

The uptake is a quantity that correlates with the concentration of iodine-avid cells within the lesion. The percentage decrease in 24-h ${ }^{124}$ I uptake between pretherapy and posttherapy imaging can be used as a quantitative measure of therapy efficacy and is referred to as the response index. A response index of $100 \%$ indicates a completely responding lesion, whereas a value of less than $100 \%$ indicates an incompletely responding lesion. For the known-volume group, the response rate was the percentage of completely responding lesions above the threshold of 85 Gy (metastases) or $300 \mathrm{~Gy}$ (TRs) in concordance with the previous studies $(2,3)$. For the small-lesion group, the response rate was assessed for each minimum-AD group and was the ratio of the number of completely responding lesions to the total number of lesions within each minimum-AD group.

The term complete response expressed the inability to distinguish a single lesion from the background; thus, the lesion may still be present but its uptake is below the detection limit of the PET imaging system. Nonvisibility of lesions by ${ }^{124}$ I PET imaging is therefore not equal to cure. The decrease of the tumor marker serum thyroglobulin, which is frequently used in quantitative response assessments, was not considered because the serum thyroglobulin value is a patient-based response quantity.

\section{Statistics}

The descriptive statistics included the mean, median, SD, minimum, and maximum. Differences between 2 groups were evaluated by the Mann-Whitney $U$ test. A $P$ value of less than 0.05 was considered to be statistically significant.

\section{RESULTS}

\section{Patient Characteristics}

Thirty-four patients met the inclusion criteria and had a total of 227 lesions, of which 59 from 17 patients yielded reliable volume estimations and 168 from 17 patients had volumes of $0.80 \mathrm{~mL}$ or less. The patients ( 22 women, 12 men) were characterized by TNM status according to the fifth edition of the AJCC Cancer Staging Manual (18) as follows: pT1, N1Mx (3 patients); N1M1 (1 patients); pT2, N1M0 (3 patients); N1Mx (2 patients); NxMx (1 patient); pT3, N1Mx (3 patients); NxM1 (1 patient); pT4, NxM1 (2 patients); N1M0 (3 patients); N1M1 (5 patients); N1Mx (7 patients); NOMx (1 patient); and NxMx (2 patients). There were 32 patients with papillary and 2 patients with follicular DTC. TSH stimulation was achieved endogenously in 30 patients of the pre- therapy group and 24 patients of the follow-up group and exogenously in 4 patients of the pretherapy group and 10 patients of the follow-up group. The descriptive statistics yielded the following values: mean age, $36 \pm 16$ y (median, $32 \mathrm{y}$; range, $16-74 \mathrm{y}$ ); single therapeutic ${ }^{131} \mathrm{I}$ activity, $8.0 \pm 4.3 \mathrm{GBq}$ (median, $10 \mathrm{GBq}$; range, 2-20 GBq); follow-up time, $6.1 \pm 3.5 \mathrm{mo}$ (median, $4.6 \mathrm{mo}$; range, $3.5-16.4 \mathrm{mo}$ ); pretherapy TSH level, $88 \pm$ $40 \mathrm{mIU} / \mathrm{mL}$ (median, $83 \mathrm{mIU} / \mathrm{mL}$; range, $30-155 \mathrm{mIU} / \mathrm{mL}$ ); and follow-up TSH level, $124 \pm 62 \mathrm{mIU} / \mathrm{mL}$ (median, $118 \mathrm{mIU} / \mathrm{mL}$; range, $41-364 \mathrm{mIU} / \mathrm{mL}$ ).

\section{Results of Known-Volume Group}

The lesions comprised 19 LMs, 10 PMs, 6 BMs, and 24 TRs. Figure 1 illustrates the statistics of the volumes, the average ADs per ${ }^{131} \mathrm{I}$ activity, and the average ADs in therapy. BM volumes were significantly larger than the other lesions. PMs showed the smallest volumes. PMs and BMs showed the lowest AD per unit activity, whereas the values were significantly greater for LMs and TRs than for PMs and BMs. The largest ADs in therapy were observed in LMs and TRs. Note that, unexpectedly, no significant difference in ADs per unit activity or in therapy were found between LMs and TRs. Of the 59 lesions, 16 responded incompletely. Five lesions (4 LMs and $1 \mathrm{BM}$ ) responded with an index of above 99\%, 10 lesions (3 LMs, 2 BMs, and 5 TRs) responded with an index of between $99 \%$ and $70 \%$, and 1 lesion (PM) showed no response (response index, $-11 \%$ ). Table 1 lists the response rates. The response rates for the different tissue types were $63 \%(12 / 19)$, $88 \%(7 / 8), 50 \%$ (3/6), and 90\% (18/20) for LMs, PMs, BMs, and TRs, respectively. Because of the notably lower response rate of LMs than of TRs, the follow-up times were inspected more closely. More than half of LMs (58\%, 11 lesions) and a quarter of TRs (25\%, 6 lesions) were imaged after a follow-up time of between 3.5 and 3.9 mo. The dose-response diagrams are illustrated in Figure 2. None of the ADs of the LMs and BMs were less than 85 Gy. Two PMs and 4 TRs were below 85 and $300 \mathrm{~Gy}$, respectively. In particular, the two completely responding metastases in the lungs received 62 and 66 Gy. Three partially responding TRs received 82,83 , and $173 \mathrm{~Gy}$, and one completely responding TR received approximately $270 \mathrm{~Gy}$.

\section{Results of Small-Volume Group}

The lesions comprised 70 LMs, 24 PMs, and 74 TRs. Figure 3 illustrates the statistics of the minimum AD per administered activity and the minimum AD in therapy. The medians of the minimum $\mathrm{AD}$ per unit activity and minimum $\mathrm{AD}$ in therapy of PMs
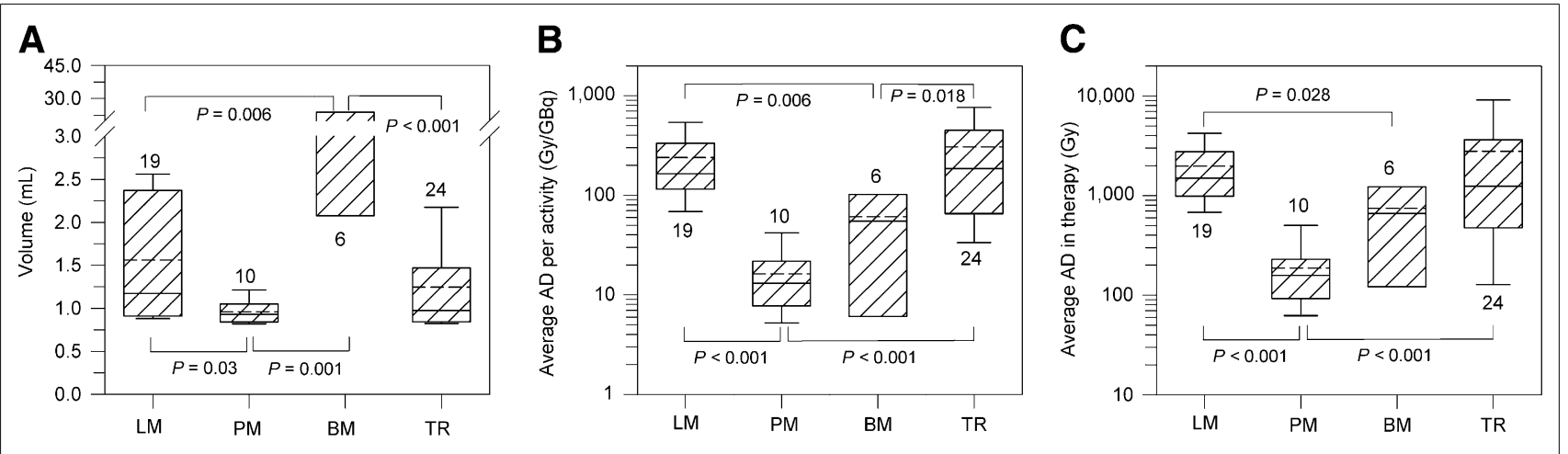

FIGURE 1. Box plots of volume (A), lesion $A D$ per ${ }^{131}$ I activity $(B)$, and predicted $A D$ in therapy $(C)$ for lesions in known-volume group. Significant differences were characterized by $P$ value below 0.05 . Numbers shown close to whiskers are numbers of lesions. 
TABLE 1

Response Rates of Known-Volume Group

\begin{tabular}{lcccc}
\hline \multicolumn{1}{c}{ Study } & LM & PM & BM $^{*}$ & TR \\
\hline Present study & $63 \%(19)$ & $88 \%(10)$ & $50 \%(6)$ & $90 \%(24)$ \\
Maxon et al. (3) & $81 \%$ & - & - & $86 \%$ \\
& & & & \\
\hline *Value was formally calculated. \\
Numbers of lesions are within parentheses.
\end{tabular}

and LMs were significantly lower than that of TRs. Approximately one tenth of the LMs and TRs were evaluated in a follow-up time of 3.5-3.9 mo, or more precisely, 9 LMs (13\%) and 7 TRs (10\%). Of the 168 lesions, 15 responded incompletely; all 24 PMs responded completely. The dose-response diagrams for the different tissue types are illustrated in Figure 4. Three lesions (1 LM and 2 TRs) exhibited a response index of more than $99 \%$, and 11 lesions showed a response index of 50\%-98\% (9 LMs and 2 TRs). One $\mathrm{LM}$ received a minimum $\mathrm{AD}$ of $7 \mathrm{~Gy}$, and its response index of $-174 \%$ clearly indicated unsuccessful treatment. Table 2 lists the response rates for the 3 minimum-AD groups, which ranged from $82 \%$ to $88 \%$ for LMs and $91 \%$ to $100 \%$ for TRs. In the minimum-high-AD group, 7 LMs and 3 TRs responded incompletely. Of those lesions, $1 \mathrm{LM}$ and 2 TRs responded nearly completely (response index, $>99 \%$ ), attaining a high minimum AD of 0.9-5.5 kGy, and were assessed in short follow-up times of 3.53.7 mo. In the minimum-low- and minimum-mid-AD groups, 3 LMs and only 1 TR responded partially. The lowest minimum $\mathrm{AD}$ of 6 Gy was observed for an LM and a TR; both lesions responded completely.

\section{DISCUSSION}

After initial radioiodine treatment, almost 90\% (196/227) of the total lesions were no longer evident on follow-up ${ }^{124}$ I PET imaging. The median response index of the 31 incompletely responding lesions was $97 \%$, and three quarters of those lesions showed a response index of more than $88 \%$, indicating considerable therapeutic efficacy. These data demonstrate that radioiodine therapy of DTC is highly effective.

\section{Response Assessment of Known-Volume Group}

Most of the lesions evaluated in the known-volume group were LMs (19) and TRs (24). For TRs, the response rate was $90 \%$ and matched well with Maxon's values, whereas the response rate for LMs was only $63 \%$ (Table 1). Besides the limited number of lesions, there are 2 effects that may explain the difference between the response rates of LMs. First, the average activity concentrations of the 7 LMs in follow-up ${ }^{124}$ I PET images at $24 \mathrm{~h}$ ranged from 0.4 to $2.8 \mathrm{kBq} / \mathrm{mL}$. Maxon et al. (2) reported that lesions with activity concentrations of approximately $15 \mathrm{kBq} / \mathrm{mL}$ were clearly detectable. Certainly, it is not anticipated that all of these incompletely responding LMs can be observed using a scintigraphic system. Therefore, the discrepancy of the response rates of LMs was, in part, caused by the sensitivity difference between PET and planar scintigraphy systems. Second, the follow-up times in the study by Maxon et al. (3) were approximately at least 10 mo. Thus, we conjecture that the difference in the response rates is additionally caused by a delayed cell-death effect of LMs, which received high ADs. In particular, 4 LMs responded nearly completely (response index, $\geq 99 \%$ ), received $1 \mathrm{kGy}$ or more, and were evaluated in a short follow-up time of 3.5-3.8 mo. This conjecture is further supported by the fact that DTC metastases are slow-growing tumors and that damaged cells can survive for months whereas radiation-induced cell death takes various routes, such as apoptosis and mitotic death.

The dose-response diagrams are shown in Figure 2. An LM with the lowest response index of $87 \%$ was observed at approximately $240 \mathrm{~Gy}$. This single partially responding lesion indicates that an $\mathrm{AD}$ of $85 \mathrm{~Gy}$ is too low; however, this conclusion cannot be justified by a single data point. The ADs of only 2 PMs and 4 TRs were below the AD thresholds. A strict application of Maxon's AD thresholds would mean that these 6 lesions are likely to be visible on follow-up imaging. Such was the case for 3 TRs with ADs ( $<180 \mathrm{~Gy}$ ), whereas one completely responding TR received an AD of $270 \mathrm{~Gy}$, close to $300 \mathrm{~Gy}$. This observation suggests that Maxon's AD threshold for TRs appears to be adequate. The ADs of the 2 completely responding PMs with volumes slightly larger than $0.80 \mathrm{~mL}$ were $60-70 \mathrm{~Gy}$, and these values were most likely underestimated (see below). Thus, the responses of the 7 lesions demonstrated that Maxon's AD thresholds were not in obvious disagreement with the results of the present study, but it did not confirm them either.
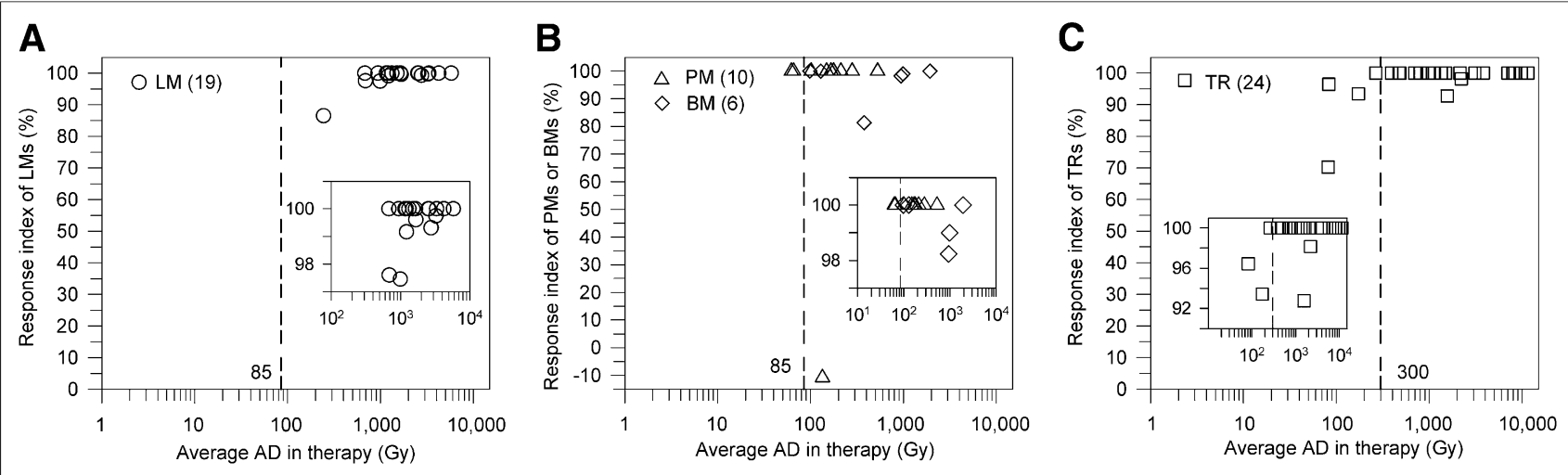

FIGURE 2. Dose-response diagrams of known-volume group for LMs (A), PMs and BMs (B), and TRs (C). Insets are enlarged views to better identify data points that are close to $100 \%$. Vertical dotted lines indicate AD thresholds for metastases (A and B) and TRs (C). Numbers within parentheses are numbers of lesions. 


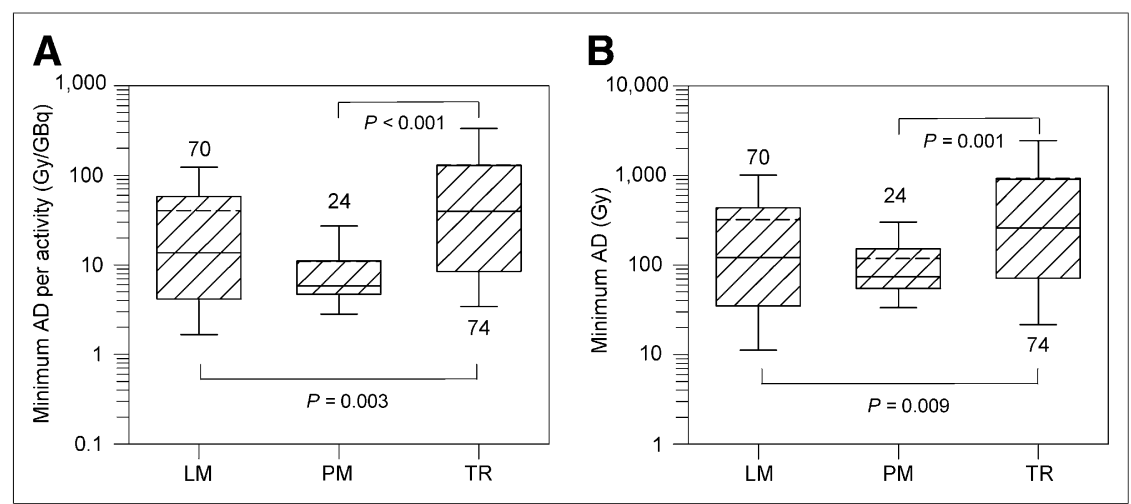

FIGURE 3. Box plots of minimum $A D$ per ${ }^{131}$ I activity $(A)$ and minimum $A D$ in therapy $(B)$ for lesions in small-volume group. Significant differences were characterized by $P$ value below 0.05 . Numbers shown close to whiskers are numbers of lesions.

Figure 1B shows the box plots of the AD per ${ }^{131}$ I activity for each tissue type. It is generally expected that tumor cells have lower radioiodine uptake capability than normal thyroid tissues, and therefore, the AD per unit activity is anticipated to be lower for metastases than for TRs. This expectation could be partially confirmed in the present study: PMs and BMs showed lower ADs per unit activity than TRs. However, a nonsignificant difference in the AD per unit activity was found for LMs and TRs; this is an unexpected finding that needs to be investigated in a larger number of lesions.

A total of 10 PMs and 6 BMs were evaluated. The ADs of PMs were underestimated for two main reasons. Respiratory motion in the lung blurred the activity and made the size of PMs appear larger in the image (19). Another reason was the increased positron range in lung tissue (20), resulting in an additional overestimation of the functional volume. An observed single PM of $0.9 \mathrm{~mL}$ yielded a response index of $-10 \%$. The almost-unchanged uptake value on follow-up ${ }^{124}$ I PET images remained unresolved for this specific PM even after the patient had received an AD of approximately $130 \mathrm{~Gy}$. Because of the low number of BMs, no decisive conclusion could be performed for this group. Nevertheless, some points are worth mentioning. The $\mathrm{AD}$ of the 3 completely responding lesions ranged from $100 \mathrm{~Gy}$ to $1.9 \mathrm{kGy}$. Two of these lesions (100-130 Gy) received ADs close to $85 \mathrm{~Gy}$, demonstrating that the AD threshold of BMs may be similar to that of LMs. It is anticipated that the response of a large BM is poor primarily because of nonuniform activity distribution. Compared with the distribution in soft tissue, the resulting nonuniform $\mathrm{AD}$ distribution is further enhanced by the higher attenuation of $\beta$ particles of ${ }^{131} \mathrm{I}$ in bone tissue. This expectation was confirmed by one partial responder

TABLE 2

Response Rates of Small-Volume Group

\begin{tabular}{lccc}
\hline & \multicolumn{3}{c}{ Type of tissue } \\
\cline { 2 - 4 } Minimum-AD group & LM & PM & TR \\
\hline Low-AD & $83 \%(6)$ & - & $100 \%(9)$ \\
Mid-AD & $88 \%(26)$ & $100 \%(13)$ & $97 \%(31)$ \\
High-AD & $82 \%(38)$ & $100 \%(11)$ & $91 \%(34)$ \\
\hline
\end{tabular}

Numbers of lesions are within parentheses. (response index, 81\%). This lesion received $380 \mathrm{~Gy}$, was $6 \mathrm{~mL}$, and showed a nonuniform activity distribution by visual inspection. The largest lesion evaluated in this study (43 mL), which also exhibited a nonuniform activity distribution, most likely responded completely because of the high average AD of $1.9 \mathrm{kGy}$ delivered. Moreover, there were 2 BMs with an AD of approximately $1 \mathrm{kGy}$; however, they did not respond completely. Their response indices were both higher than $98 \%$, demonstrating a considerable therapeutic efficacy. Again, a delayed complete response might also occur for BMs as suggested for LMs. This possibility is supported by the relatively short follow-up time of $4.2 \mathrm{mo}$.

\section{Response Assessment of Small-Volume Group}

There is a general need for an AD approach for small lesions because approximately three quarters of the total lesions (168/227) in 34 DTC patients were too small; that is, they were below the lower determinability limit of the applied volume segmentation method. A dosimetry methodology used by Furhang et al. (21) and Maxon (22) was adapted to estimate the minimum ADs of these small lesions. They used a fixed volume of 1 and $0.15 \mathrm{~mL}$, respectively. In our study, a fixed sphere volume of $0.80 \mathrm{~mL}$ was used to calculate the minimum ADs.

This discussion has focused mainly on the response assessment of LMs and TRs. Maxon's response rates matched well with those of the minimum-high-AD group in this study, namely $82 \%$ for LMs and $91 \%$ for TRs (Table 2). This study therefore verifies Maxon's historical data. For comparison, the results of the TR response rate of $90 \%$ determined for the known-volume group also matched well that of the minimum-high-AD group. In contrast, the LM response rate was only $63 \%$ in the known-volume group and deviated from the results found in the minimum-high-AD group. In the light of the findings in the small-volume group, the low LM response rate of $63 \%$ is most likely attributable to too short a followup time (3.5-3.9 mo) for $60 \%$ of the LMs in the known-volume group, whereas only one tenth of the LMs in the small-volume group was evaluated between 3.5 and 3.9 mo after radioiodine therapy. Thus, the results of the response rate comparison between the 2 lesion groups corroborated that a delayed cell-death effect may be the primary reason for the observed LM response rate discrepancy between the known- and small-volume groups.

A striking finding of this study was that the response rates in the minimum-low- and minimum-mid-AD groups were relatively high, ranging from $83 \%$ to $100 \%$ (Table 2). Even a minimum AD of 6 Gy observed for an LM and a TR yielded a complete response. Hence, we concluded that a minimum AD of approximately 10 Gy or more for both LMs and TRs is associated with high response rates; this finding requires a cogent explanation.

The similarity of the response rates for all minimum-AD groups suggests that the actual ADs in the minimum-low- and minimummid-AD groups are at least $85 \mathrm{~Gy}$ for LMs or at least $300 \mathrm{~Gy}$ for TRs. Those actual ADs can be achieved only if the diameters of most of the lesions evaluated in the minimum-low- and minimummid-AD groups are considerably smaller than $11.5 \mathrm{~mm}$. Supplemental Table 1 lists the minimum ADs that are theoretically needed to yield an average AD of 85 Gy for metastases or $300 \mathrm{~Gy}$ for TRs as a function of the actual lesion diameters. The theoretic 

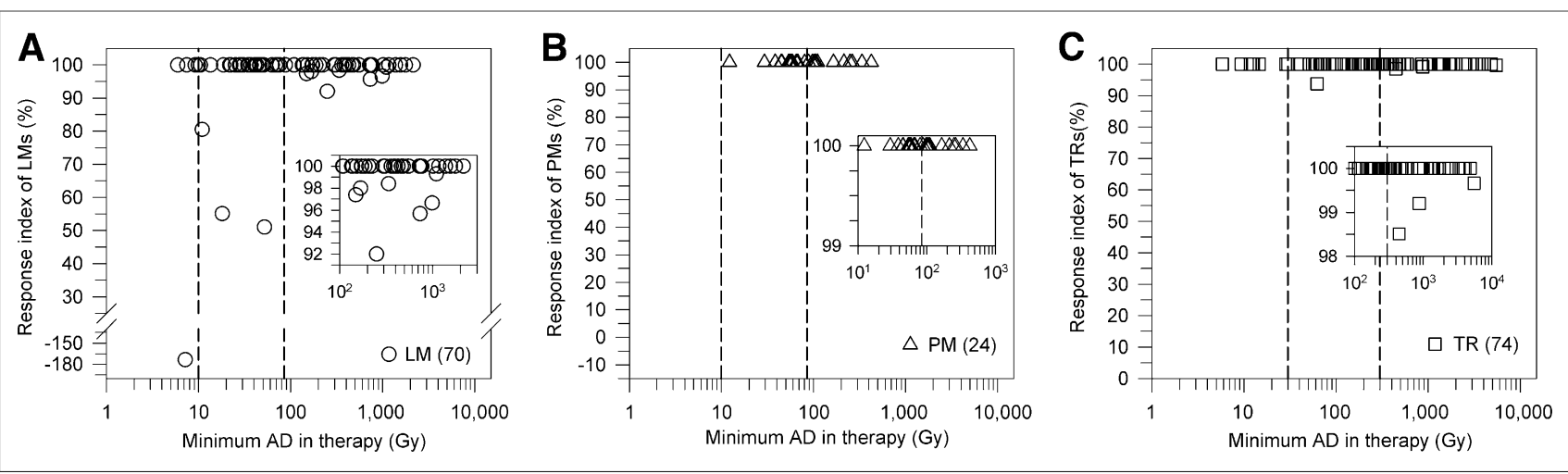

FIGURE 4. Dose-response diagrams of small-volume group for LMs (A), PMs (B), and TRs (C). Dashed lines indicate dividing lines for separating different minimum-AD groups. Insets are enlarged views to better identify data points that are close to $100 \%$. Vertical dotted lines indicate $A D$ thresholds for metastases (A and B) and TRs (C). Numbers within parentheses are numbers of lesions.

derivation (described in the supplemental materials available online at http://jnm.snmjournals.org) takes into account the partialvolume and particle range effects. In particular, lesion-diameter ranges for LMs and TRs of approximately 4.0-5.0 and 2.5-4.5 $\mathrm{mm}$, respectively, in the minimum-low-AD group and 5.0-11.5 and $4.5-11.5 \mathrm{~mm}$, respectively, in the minimum-mid-AD group produced actual $\mathrm{AD}$ estimations of at least 85 and $300 \mathrm{~Gy}$, respectively. The calculated diameter range of 4.0-11.5 mm for LMs overlaps well with the basic diameter range of $3-15 \mathrm{~mm}$ for normal lymph nodes in the head-neck region (23). In addition, the finding in this study that approximately three quarters of the LMs evaluated were smaller than $11.5 \mathrm{~mm}$ also agreed well with findings in the basic literature-that is, that approximately $75 \%$ of normal lymph nodes in the head-neck region are smaller than $10 \mathrm{~mm}$. Thus, most LMs and TRs evaluated in the minimum-lowand minimum-mid-AD groups may have been notably smaller than $11.5 \mathrm{~mm}$, thus explaining their high response rates and accounting for the high percentage of lesions that were not amenable to reliable volume estimations.

For all 24 PMs, a complete response was observed (Fig. 4B). Approximately half of the PMs were localized in each of the 2 groups: minimum-mid and minimum-high $\mathrm{AD}$. The minimum ADs ranged from 12 to $424 \mathrm{~Gy}$. The observation of a response rate of $100 \%$ implies that the actual ADs are notably larger than the minimum ADs. Again, one obvious explanation is that the actual diameters of the 24 PMs are considerably smaller than $11.5 \mathrm{~mm}$, but respiratory motion-related blurring and positron range effects also further enhance the $\mathrm{AD}$ underestimation $(19,20)$.

Often, standard activities of 3.7-7.4 GBq are used for DTC patients (4). In contrast, individualized therapy activities are administered on the basis of the results of the patient-specific tracer dosimetry before a planned radioiodine therapy, including both lesion and organ-at-risk dosimetry (6). Because of the time and effort required for the ${ }^{124} \mathrm{I}$ PET/CT dosimetry approach and the availability of ${ }^{124} \mathrm{I}$, the standard-activity approach most likely remains the basic concept in most institutions and will not change in the near future. One reason is that the standard-activity approach has proven to be highly effective, mostly without exceeding the toxicity levels of the organs at risk. On the basis of the results found in this study, we conjecture that the high therapeutic efficacy of standard activities is related to the fact that most of the lesions in the initial radioiodine treatment were relatively small and showed a relatively high radioiodine uptake. For small lesions, a complete response can be expected even for minimum ADs of approximately $10 \mathrm{~Gy}$ - a minimum-AD threshold that can be used in future pretherapy ${ }^{124}$ I PET/CT dosimetry based on the methodology of this study.

\section{CONCLUSION}

Radioiodine therapy of DTC is highly effective. For the knownvolume group, the PET-based response rate for TRs matched well that of the historical data derived using planar ${ }^{131}$ I scintigraphy, whereas the response rate for LM was not as high as expected. A reasonable explanation for the deviation in the LM response rates is most likely too short a follow-up time and, in part, the higher sensitivity of PET imaging. The response rates of small lesions were independent of the minimum ADs, suggesting that a high proportion of the small lesions was considerably smaller than $11.5 \mathrm{~mm}$. Finally, the PET-based response rates of small LMs and TRs in the minimum-high-AD dose group matched the historical data well.

\section{DISCLOSURE}

The costs of publication of this article were defrayed in part by the payment of page charges. Therefore, and solely to indicate this fact, this article is hereby marked "advertisement" in accordance with 18 USC section 1734 . No potential conflict of interest relevant to this article was reported.

\section{REFERENCES}

1. Durante C, Haddy N, Baudin E. Long-term outcome of 444 patients with distant metastases from papillary and follicular thyroid carcinoma: benefits and limits of radioiodine therapy. J Clin Endocrinol Metab. 2006;91:2892-2899.

2. Maxon HR, Thomas SR, Hertzberg VS, et al. Relation between effective radiation dose and outcome of radioiodine therapy for thyroid cancer. $N$ Engl J Med. 1983;309:937-941.

3. Maxon HR, Englaro EE, Thomas SR, et al. Radioiodine-131 therapy for welldifferentiated thyroid cancer: quantitative radiation dosimetric approach-outcome and validation in 85 patients. J Nucl Med. 1992;33:1132-1136.

4. Luster M, Clarke SE, Dietlein M, et al. Guidelines for radioiodine therapy of differentiated thyroid cancer. Eur J Nucl Med Mol Imaging. 2008;5:1941-1959.

5. Cooper DS, Doherty GM, Haugen BR, et al. Revised American Thyroid Association management guidelines for patients with thyroid nodules and differentiated thyroid cancer. Thyroid. 2009;19:1167-1214.

6. Freudenberg LS, Jentzen W, Görges R, et al. ${ }^{124}$ I-PET dosimetry in advanced differentiated thyroid cancer: therapeutic impact. Nuklearmedizin. 2007;46: 121-128. 
7. Capoccetti F, Criscuoli B, Rossi G, et al. The effectiveness of ${ }^{124}$ I PET/CT in patients with differentiated thyroid cancer. Q J Nucl Med Mol Imaging. 2009; 53:536-545.

8. Hobbs RF, Wahl RL, Lodge MA, et al. ${ }^{124}$ I PET-based 3D-RD dosimetry for a pediatric thyroid cancer patient: real-time treatment planning and methodologic comparison. J Nucl Med. 2009;50:1844-1847.

9. de Pont C, Halders S, Bucerius J, et al. ${ }^{124} \mathrm{I}$ PET/CT in the pretherapeutic staging of differentiated thyroid carcinoma: comparison with posttherapy ${ }^{131}$ I SPECT/CT. Eur J Nucl Med Mol Imaging. 2013;40:693-700.

10. Jentzen W, Freudenberg L, Bockisch A. Quantitative imaging of ${ }^{124} \mathrm{I}$ with PET/CT in pretherapy lesion dosimetry: effects impairing image quantification and their corrections. Q J Nucl Med Mol Imaging. 2011;55:21-43.

11. Jentzen W, Freudenberg LS, Heinze M, et al. Segmentation of PET volumes by iterative image thresholding. J Nucl Med. 2007;48:108-114.

12. Rosenbaum-Krumme S, Nagarajah J, Ruhlmann M, et al. ${ }^{124}$ I-PET/CT images of differentiated thyroid cancer patients: distinguishing lymph node metastases from thyroid remnants using kinetic quantities. Nuklearmedizin. 2012;51:213-216.

13. Dietlein M, Dressler J, Eschner W, et al. Procedure guideline for iodine-131 whole-body scintigraphy for differentiated thyroid cancer (version 3). Nuklearmedizin. 2007;46:206-212.

14. Jentzen W, Freudenberg L, Eising EG, et al. Optimized ${ }^{124}$ I PET dosimetry protocol for radioiodine therapy of differentiated thyroid cancer. $\mathrm{J} \mathrm{Nucl} \mathrm{Med}$. 2008;49:1017-1023.
15. Jentzen W. Experimental investigation of factors affecting the absolute recovery coefficients in iodine-124 PET lesion imaging. Phys Med Biol. 2010;55:23652398.

16. Jentzen W, Hobbs R, Stahl A, et al. Pre-therapeutic iodine-124 PET(/CT) dosimetry confirms low average absorbed doses per administered iodine-131 activity to the salivary glands in radioiodine therapy of differentiated thyroid cancer. Eur J Nucl Med Mol Imaging. 2010;37:884-895.

17. McDougall IR, Iagaru A. Thyroid stunning: fact or fiction? Semin Nucl Med. 2011;41:105-112.

18. Fleming ID, Cooper JS, Henson DE, et al., eds. AJCC Cancer Staging Manual. 5th ed. Philadelphia, PA: Lippincott-Raven; 1997.

19. Pevsner A, Nehmeh SA, Humm JL, et al. Effect of motion on tracer activity determination in CT attenuation corrected PET images: a lung phantom study. Med Phys. 2005;32:2358-2362.

20. Kemerink GJ, Visser MG, Franssen R, et al. Effect of the positron range of ${ }^{18} \mathrm{~F}$, ${ }^{68} \mathrm{Ga}$ and ${ }^{124} \mathrm{I}$ on PET/CT in lung-equivalent materials. Eur J Nucl Med Mol Imaging. 2011;38:940-948.

21. Furhang EE, Larson SM, Buranapong P, et al. Thyroid cancer dosimetry using clearance fitting. J Nucl Med. 1999;40:131-136.

22. Maxon HR. Quantitative radioiodine therapy in the treatment of differentiated thyroid cancer. Q J Nucl Med. 1999;43:313-323.

23. Feyerabend T, Richter E, eds. Normal Lymph Node Topography: CT-Atlas. 2nd ed. New York, NY: Springer-Verlag; 2004:7. 